This is a self-archived version of an original article. This version may differ from the original in pagination and typographic details.

Author(s): Koskela, Pekka; Wang, Zhuang; Xu, Haiqing

Title: Controlled diffeomorphic extension of homeomorphisms

Year: 2018

Version: Accepted version (Final draft)

Copyright: @ 2018 Elsevier Ltd

Rights: $C C B Y-N C-N D 4.0$

Rights url: https://creativecommons.org/licenses/by-nc-nd/4.0/

Please cite the original version:

Koskela, P., Wang, Z., \& Xu, H. (2018). Controlled diffeomorphic extension of homeomorphisms. Nonlinear Analysis : Theory, Methods and Applications, 177(Part B), 586-600.

https://doi.org/10.1016/j.na.2018.04.020 


\title{
Controlled diffeomorphic extension of homeomorphisms
}

\author{
Pekka Koskela, Zhuang Wang, and Haiqing Xu
}

\begin{abstract}
Let $\Omega$ be an internal chord-arc Jordan domain and $\varphi: \mathbb{S} \rightarrow \partial \Omega$ be a homeomorphism. We show that $\varphi$ has finite dyadic energy if and only if $\varphi$ has a diffeomorphic extension $h: \mathbb{D} \rightarrow \Omega$ which has finite energy.
\end{abstract}

Keywords: Poisson extension, diffeomorphism, chord-arc curve.

\section{Introduction}

Let $\Omega \subset \mathbb{C}$ be a bounded convex domain and suppose that $\varphi$ is a homeomorphism from the unit circle $\mathbb{S}$ onto $\partial \Omega$. Then, by [7], the complex-valued Poisson extension $h$ of $\varphi$ is a homeomorphism from $\overline{\mathbb{D}}$ onto $\bar{\Omega}$. This harmonic map $h$ is a diffeomorphism in $\mathbb{D}$ but its derivatives are not necessarily uniformly bounded. In 2007, G. C. Verchota [10] proved that the derivatives of $h$ may fail to be square integrable but that they are necessarily $p$-integrable over $\mathbb{D}$ for any $p<2$. In 2009 , T. Iwaniec, $\mathrm{G}$. Martin and C. Sbordone improved on [6] by showing that the derivatives belong to weak- $L^{2}$ with sharp estimates. In a related work [1] by K. Astala, T. Iwaniec, G. Martin and J. Onninen, it was shown that if additionally $\partial \Omega$ is a $C^{1}$-regular Jordan curve, the square integrability of the derivatives of $h$ is equivalent to the requirement that

$$
\int_{\partial \Omega} \int_{\partial \Omega}|\log | \varphi^{-1}(\xi)-\varphi^{-1}(\eta)|||d \xi||d \eta|<+\infty .
$$

In this note we give a generalization of the aforementioned results. Towards this end, recall that the Poisson extension of a homeomorphism $\varphi: \mathbb{S} \rightarrow \partial \Omega$ may fail to be injective if $\Omega$ is not convex. Next, the boundary $\partial \Omega$ of a bounded convex domain $\Omega$ is a chord-arc Jordan curve: $\partial \Omega$ is a rectifiable Jordan curve and there is a constant $C$ such that for all $w_{1}, w_{2} \in \partial \Omega$,

$$
\ell\left(w_{1}, w_{2}\right) \leq C\left|w_{1}-w_{2}\right|,
$$

where $\ell\left(w_{1}, w_{2}\right)$ is the arc length of the shorter arc of $\partial \Omega$ joining $w_{1}$ to $w_{2}$. A domain whose boundary is a chord-arc Jordan curve is called a chord-arc Jordan domain. 
Hence we are lead to ask for the optimal regularity of homeomorphic extensions $h: \mathbb{D} \rightarrow \Omega$ for a given homeomorphism $\varphi: \mathbb{S} \rightarrow \partial \Omega$ and a chord-arc Jordan domain $\Omega$.

Before introducing our first result, let us fix a dyadic decomposition $\left\{\Gamma_{j, k}: j \in\right.$ $\left.\mathbb{N}, k=1, \cdots, 2^{j}\right\}$ of $\mathbb{S}$, such that for a fixed $j \in \mathbb{N},\left\{\Gamma_{j, k}: k=1, \cdots, 2^{j}\right\}$ is a family of arcs of length $2 \pi / 2^{j}$ with $\bigcup_{k} \Gamma_{j, k}=\mathbb{S}$. The next generation is constructed in such a way that for each $k \in\left\{1, \cdots, 2^{j+1}\right\}$, there exists a unique number $k^{\prime} \in\left\{1, \cdots, 2^{j}\right\}$, satisfying $\Gamma_{j+1, k} \subset \Gamma_{j, k^{\prime}}$. Here, we call $\Gamma_{j, k^{\prime}}$ the parent of $\Gamma_{j+1, k}$. Fix $\Gamma_{1,1}$ to be the image of $[0, \pi]$ under the map $\theta \mapsto e^{i \theta}$. We denoted by $\ell\left(\varphi\left(\Gamma_{j, k}\right)\right)$ the arc length of the image arc of $\Gamma_{j, k}$ under the homeomorphism $\varphi$.

Theorem 1. Let $\Omega \subset \mathbb{C}$ be an internal chord-arc Jordan domain and suppose that $\varphi: \mathbb{S} \rightarrow \partial \Omega$ is a homeomorphism. Then for $\lambda \in(-1,+\infty)$, the following are equivalent:

(i) $\int_{\mathbb{D}}|D h(z)|^{2} \log ^{\lambda}(e+|D h(z)|) d z<+\infty$ for some diffeomorphic extension $h$ : $\mathbb{D} \rightarrow \Omega$ of $\varphi$;

(ii) $\int_{\mathbb{D}}|D h(z)|^{2} \log ^{\lambda}\left(\frac{2}{1-|z|}\right) d z<+\infty$ for some diffeomorphic extension $h: \mathbb{D} \rightarrow \Omega$ of $\varphi$;

(iii) $\int_{\partial \Omega} \int_{\partial \Omega}|\log | \varphi^{-1}(\eta)-\left.\varphi^{-1}(\xi)\right|^{\lambda+1}|d \eta||d \xi|<+\infty$;

(iv) $\sum_{j=1}^{\infty} j^{\lambda} \sum_{k=1}^{2^{j}} \ell\left(\varphi\left(\Gamma_{j, k}\right)\right)^{2}<+\infty$;

(v) $\sum_{j=1}^{\infty} \sum_{k=1}^{2^{j}} \ell\left(\varphi\left(\Gamma_{j, k}\right)\right)^{2} \log ^{\lambda}\left(e+\frac{\ell\left(\varphi\left(\Gamma_{j, k}\right)\right)}{2^{-j}}\right)<+\infty$.

A Jordan domain $\Omega \subset \mathbb{C}$ is an internal chord-arc Jordan domain if $\partial \Omega$ is rectifiable and there is a constant $C>0$ such that for all $w_{1}, w_{2} \in \partial \Omega$,

$$
\ell\left(w_{1}, w_{2}\right) \leq C \lambda_{\Omega}\left(w_{1}, w_{2}\right)
$$

where $\ell\left(w_{1}, w_{2}\right)$ is the arc length of the shorter arc of $\partial \Omega$ joining $w_{1}$ to $w_{2}$ and $\lambda_{\Omega}\left(w_{1}, w_{2}\right)$ is the internal distance between $w_{1}, w_{2}$, which is defined as

$$
\lambda_{\Omega}\left(w_{1}, w_{2}\right)=\inf _{\alpha} \ell(\alpha)
$$

where the infimum is taken over all rectifiable arcs $\alpha \subset \Omega$ joining $w_{1}$ and $w_{2}$; if there is no rectifiable curve joining $w_{1}$ and $w_{2}$, we let $\lambda_{\Omega}\left(w_{1}, w_{2}\right)=\infty$; cf. [9, Section 3.1] or [2, Section 2].

Naturally, every chord-arc Jordan domain is also an internal chord-arc domain, but there are internal chord-arc domains that fail to be chord-arc; e.g., the inward cusp domain:

$$
\Omega_{\epsilon}=\mathbb{D} \backslash\left\{(x, y) \in \mathbb{R}^{2}: 0 \leq x \leq 1,|y| \leq x^{2}\right\} .
$$

The statement of Theorem 1 does not allow for $\lambda \leq-1$. Actually, (i)-(v) all hold for $\lambda<-1$, independently of $\varphi$. When $\lambda=-1$, (iii) needs to be reformulated via a 
double logarithm, after which one still has a list of mutually equivalent conditions that may or may not hold, see [11.

In the case when $\Omega=\mathbb{D}$, Theorem 1 can be formulated via the harmonic Poisson extension.

Theorem 2. Let $\varphi: \mathbb{S} \rightarrow \mathbb{S}$ be a homeomorphism and $h=P[\varphi]: \mathbb{D} \rightarrow \mathbb{D}$ be the harmonic Poisson extension of $\varphi$. Then for $\lambda \in(-1,+\infty)$ the following are equivalent:

(i) $\int_{\mathbb{D}}|D h(z)|^{2} \log ^{\lambda}(e+|D h(z)|) d z<+\infty$;

(ii) $\int_{\mathbb{D}}|D h(z)|^{2} \log ^{\lambda}\left(\frac{2}{1-|z|}\right) d z<+\infty$;

(iii) $\int_{\mathbb{S}} \int_{\mathbb{S}}|\log | \varphi^{-1}(\eta)-\varphi^{-1}(\xi)||^{\lambda+1}|d \eta||d \xi|<+\infty$;

(iv) $\sum_{j=1}^{\infty} j^{\lambda} \sum_{k=1}^{2^{j}} \ell\left(\varphi\left(\Gamma_{j, k}\right)\right)^{2}<+\infty$;

(v) $\sum_{j=1}^{\infty} \sum_{k=1}^{2^{j}} \ell\left(\varphi\left(\Gamma_{j, k}\right)\right)^{2} \log ^{\lambda}\left(e+\frac{\ell\left(\varphi\left(\Gamma_{j, k}\right)\right)}{2^{-j}}\right)<+\infty$.

Our main task is actually to prove Theorem 2. Indeed, once we know that Theorem 2 holds, Theorem 1 is obtained via a suitable change of variable, relying on the fact that there is a bi-Lipschitz map from $\overline{\mathbb{D}}$ onto $\left(\bar{\Omega}, \lambda_{\Omega}\right)$; see Section 3.2 .

The paper is organized as follows. In Section 2 we give some relevant facts about the dyadic decomposition of $\mathbb{S}$ and some properties of the $N$-function $\Phi(t)=$ $t^{2} \log ^{\lambda}(e+t)$ for $\lambda>-1$. Section 3 contains the full proofs of Theorem 1 and Theorem 2.

\section{Preliminaries}

\subsection{Dyadic decomposition}

Since our dyadic decomposition $\left\{\Gamma_{j, k}: j \in \mathbb{N}, k=1, \cdots, 2^{j}\right\}$ of $\mathbb{S}$ satisfies that $\Gamma_{1,1}$ is the image of $[0, \pi]$ under the map $\theta \mapsto e^{i \theta}$, we may assume that the dyadic decomposition $\left\{I_{j, k}=\left[2 \pi(k-1) / 2^{j}, 2 \pi k / 2^{j}\right]: j \in \mathbb{N}, k=1, \cdots, 2^{j}\right\}$ of the interval $[0,2 \pi]$ matches with $\left\{\Gamma_{j, k}\right\}$ in the sense that each $\Gamma_{j, k}$ is exactly the image of $I_{j, k}$ under the map $\theta \mapsto e^{i \theta}$. Moreover, the dyadic arc $\Gamma_{j, k}$ is called a $j$-level dyadic arc, and we denote the two end points of $\Gamma_{j, k}$ by $\xi_{j, k}$ and $\xi_{j, k+1}$. Two dyadic arcs which are $j$-level dyadic arcs for some $j \in \mathbb{N}$ are called brother dyadic arcs if they have a common parent.

For the dyadic decompositions $\left\{\Gamma_{j, k}\right\}$ of $\mathbb{S}$ and $\left\{I_{j, k}\right\}$ of $[0,2 \pi]$ as above, we have a decomposition of the unit disk $\mathbb{D}$ given by $\left\{Q_{j, k}: j \in \mathbb{N}, k=1, \cdots, 2^{j}\right\}$ where

$$
Q_{j, k}=\left\{r e^{i \theta}: 1-1 / 2^{j-1} \leq r \leq 1-1 / 2^{j}, \theta \in I_{j, k}\right\}
$$

for any $j \in \mathbb{N}$ and $k=1, \cdots, 2^{j}$, see Figure 1 . It is easy to see that $\operatorname{dist}\left(Q_{j, k}, \mathbb{S}\right)=2^{-j}$ and that if $P: \mathbb{D} \rightarrow \mathbb{S}$ is the radial projection map, then $P\left(Q_{j, k}\right)=\Gamma_{j, k}$ for all $j \in \mathbb{N}$ 


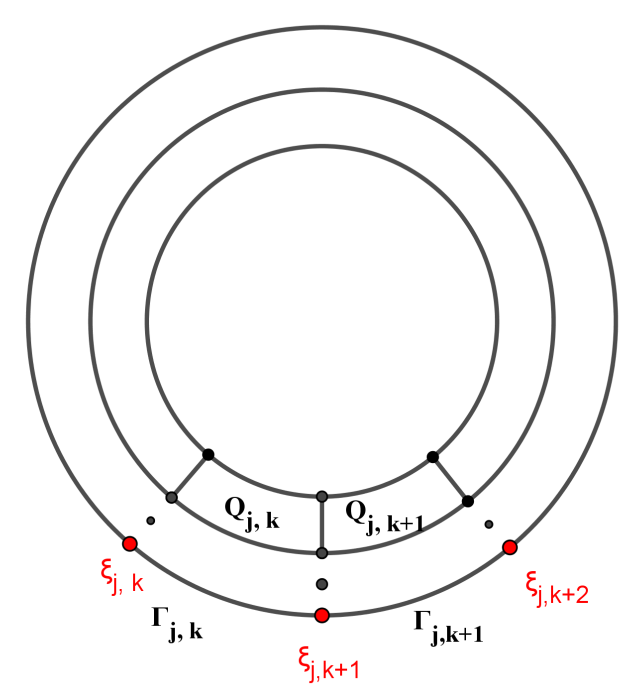

Figure 1: The decompositions of $\mathbb{S}$ and $\mathbb{D}$

and $k=1, \cdots, 2^{j}$. There is an uniform constant $C>0$ such that for any $Q_{j, k}$ with center $x_{j, k}=r_{j, k} e^{i \theta_{j, k}}, r_{j, k}=1-3 / 2^{j+1}$ and $\theta_{j, k}=\pi(2 k-1) / 2^{j}$, we have

$$
B\left(x_{j, k}, C^{-1} \operatorname{diam}\left(Q_{j, k}\right)\right) \subset Q_{j, k} \subset B\left(x_{j, k}, C \operatorname{diam}\left(Q_{j, k}\right)\right) .
$$

So for any $Q_{j, k}$, we can find a disk $B_{j, k}$ satisfying $B_{j, k} \subset Q_{j, k} \subset C B_{j, k}$, where $C$ is a constant independent of $Q_{j, k}$.

In order to later estimate the integral of $|D h|$ over $Q_{j, k}$, we employ the following decomposition of $\mathbb{S}$ via the dyadic $\operatorname{arcs} \Gamma_{i, l}$ with $i \leq j$. The idea is to build a dyadic-type annular decomposition around $\Gamma_{j, k}$.

We fix $\Gamma_{j, k}$ and construct the decomposition via the following steps. For simplicity, we assume that the unique brother arc of $\Gamma_{j, k}$ is $\Gamma_{j, k+1}$ and located at the anticlockwise side of $\Gamma_{j, k}$.

Step 1. On the anticlockwise side of $\Gamma_{j, k+1}$, we choose the unique arc $\Gamma_{j-1, m_{j-1}^{1}}$ so that $\Gamma_{j, k+1} \cap \Gamma_{j-1, m_{j-1}^{1}}$ is a singleton. If the arc $\Gamma_{j-1, m_{j-1}^{1}+1}$ shares the common parent with $\Gamma_{j-1, m_{j-1}^{1}}$, then set $\left(\Gamma_{j-1, m_{j-1}^{1}+1}\right)=\Gamma_{j-1, m_{j-1}^{1}+1}$. If not, set $\left(\Gamma_{j-1, m_{j-1}^{1}+1}\right)=\emptyset$. Then define $\Gamma_{j-1, m_{j-1}^{2}}$ and $\left(\Gamma_{j-1, m_{j-1}^{2}-1}\right)$ analogously along the clockwise side of $\Gamma_{j, k}$.

Step 2. Repeat the process in Step 1 with $\Gamma_{j, k+1}$ replaced by $\Gamma_{j-1, m_{j-1}^{1}}$ if we have $\left(\Gamma_{j-1, m_{j-1}^{1}+1}\right)=\emptyset$ or $\Gamma_{j-1, m_{j-1}^{1}+1}$ otherwise, and with $\Gamma_{j, k}$ replaced by $\Gamma_{j-1, m_{j-1}^{2}}$ if $\left(\Gamma_{j-1, m_{j-1}^{2}-1}\right)=\emptyset$ or $\Gamma_{j-1, m_{j-1}^{2}-1}$ otherwise; unless we get $\Gamma_{j_{0}, m_{j_{0}}^{1}},\left(\Gamma_{j_{0}, m_{j_{0}}^{1}+1}\right), \Gamma_{j_{0}, m_{j_{0}}^{2}}$ and $\left(\Gamma_{j_{0}, m_{j_{0}}^{2}-1}\right)$ such that

$$
\left(\Gamma_{j_{0}, m_{j_{0}}^{1}} \cup\left(\Gamma_{j_{0}, m_{j_{0}}^{1}+1}\right)\right) \bigcap\left(\Gamma_{j_{0}, m_{j_{0}}^{2}} \cup\left(\Gamma_{j_{0}, m_{j_{0}}^{2}-1}\right)\right) \neq \emptyset
$$


This procedure decomposes $\mathbb{S}$ :

$$
\begin{aligned}
\mathcal{P}\left(\Gamma_{j, k}\right)=\{ & \Gamma_{j, k}, \Gamma_{j, k+1}, \Gamma_{j-1, m_{j-1}^{1}},\left(\Gamma_{j-1, m_{j-1}^{1}+1}\right), \Gamma_{j-1, m_{j-1}^{2}},\left(\Gamma_{j-1, m_{j-1}^{2}-1}\right), \\
& \left.\ldots, \Gamma_{j_{0}, m_{j_{0}}^{1}},\left(\Gamma_{j_{0}, m_{j_{0}}^{1}}+1\right), \Gamma_{j_{0}, m_{j_{0}}^{2}},\left(\Gamma_{j_{0}, m_{j_{0}}^{2}-1}\right)\right\} .
\end{aligned}
$$

where each $(\cdot)$ is either empty or a dyadic arc. Correspondingly, we obtain a decomposition of $[0,2 \pi]$ as

$$
\begin{aligned}
\mathcal{P}\left(I_{j, k}\right)=\{ & I_{j, k}, I_{j, k+1}, I_{j-1, m_{j-1}^{1}},\left(I_{j-1, m_{j-1}^{1}+1}\right), I_{j-1, m_{j-1}^{2}},\left(I_{j-1, m_{j-1}^{2}-1}\right), \\
& \left.\ldots, I_{j_{0}, m_{j_{0}}^{1}},\left(I_{j_{0}, m_{j_{0}}^{1}+1}\right), I_{j_{0}, m_{j_{0}}^{2}},\left(I_{j_{0}, m_{j_{0}}^{2}-1}\right)\right\} .
\end{aligned}
$$

Let us illustrate this procedure by an example, see Figure 2 below. We use a binary tree to represent the dyadic decomposition $\left\{\Gamma_{j, k}\right\}$ of $\mathbb{S}$. Suppose that we are given a dyadic arc $\Gamma_{4,7}$. Then $\Gamma_{4,8}$ is the unique brother arc of $\Gamma_{4,7}$. In Step 1, we choose $\Gamma_{3,5}$ and $\Gamma_{3.6}$ on the anticlockwise side of $\Gamma_{4,8}$, and $\Gamma_{3,3}$ on the clockwise side of $\Gamma_{4,7}$. In Step 2, we choose $\Gamma_{2,4}$ on the anticlockwise side of $\Gamma_{3,6}$, and $\Gamma_{2,1}$ on the clockwise side of $\Gamma_{3,3}$. There will be no more steps because $\Gamma_{2,1} \cap \Gamma_{2,4} \neq \emptyset$.

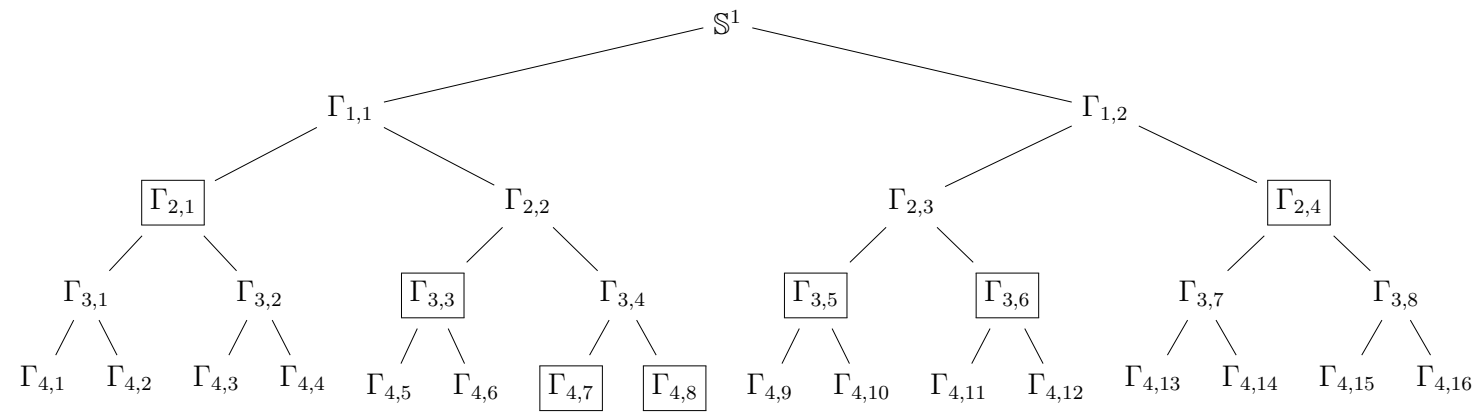

Figure 2: The decomposition of $\mathbb{S}$ around $\Gamma_{4,7}$

Suppose we are given an $n$-level arc $\Gamma_{n, m}$. We are interested in the number of $j$-level arcs, $j \geq n$, which can induce $\Gamma_{n, m}$ by the above decomposition method. Here, we say that a dyadic arc $\Gamma_{j, l}$ induces $\Gamma_{n, m}$ by the above decomposition method if and only if $\Gamma_{n, m}$ is in the set $\mathcal{P}\left(\Gamma_{j, l}\right)$.

To begin, $\Gamma_{n, m}$ can be first induced by its brother (without loss of generality assume that it is $\left.\Gamma_{n, m+1}\right)$, and two couples of $(n+1)$-level arcs. Notice that among all $(n+1)$-level arcs, there is only one couple that can induce both $\Gamma_{n, m}$ and $\Gamma_{n, m+1}$. It follows that $\Gamma_{n, m}$ can be induced by three couples of $(n+1)$-level arcs. Choose one of these $(n+1)$-level arcs. There is only one couple of $(n+2)$-level arcs which induce both $\Gamma_{n, m}$ and the chosen $(n+1)$-level arcs. Then $\Gamma_{n, m}$ can be induced by six couples of $(n+2)$-level arcs. Generally, for $j \geq n$ we have

$$
\sharp\left\{\Gamma: \Gamma \text { is a } j \text {-level dyadic arc and induce } \Gamma_{n, m}\right\} \leq 3 \cdot 2^{j-n} \text {. }
$$




\section{$2.2 \quad N$-functions}

A function $\Phi:[0, \infty) \rightarrow[0, \infty)$ is an $N$-function if it is a continuous, increasing and convex function satisfying $\Phi(0)=0$,

$$
\lim _{t \rightarrow 0+} \frac{\Phi(t)}{t}=0 \text { and } \lim _{t \rightarrow+\infty} \frac{\Phi(t)}{t}=+\infty .
$$

An $N$-function $\Phi$ can be expressed as

$$
\Phi(t)=\int_{0}^{t} \phi(s) d s,
$$

where $\phi:[0, \infty) \rightarrow[0, \infty)$ is an increasing, right-continuous function with $\phi(0)=0$ and $\lim _{t \rightarrow+\infty} \phi(t)=+\infty$.

For each $N$-function $\Phi$ and $t \geq 0$, set

$$
\psi(t)=\sup _{\phi(s) \leq t} s \text { and } \Psi(t)=\int_{0}^{t} \psi(s) d s .
$$

Then we call $\Psi$ the complementary function of $\Phi$. The complementary function of an $N$-function is also an $N$-function. We call $\Psi, \Phi$ a pair of complementary $N$-functions.

An $N$-function $\Phi$ is said to satisfy the $\Delta_{2}$-condition if there is a constant $C_{\Phi}>0$, called a doubling constant of $\Phi$, such that

$$
\Phi(2 t) \leq C_{\Phi} \Phi(t), \forall t \geq 0 .
$$

Proposition 1. If an $N$-function $\Phi$ satisfies the $\Delta_{2}$-condition, then for any constant $c>0$, there exist $c_{1}, c_{2}>0$ such that

$$
c_{1} \Phi(t) \leq \Phi(c t) \leq c_{2} \Phi(t) \quad \text { for all } \quad t \geq 0
$$

where $c_{1}$ and $c_{2}$ depend only on $c$ and the doubling constant $C_{\Phi}$. Therefore, we obtain that if $A \approx B$, then $\Phi(A) \approx \Phi(B)$.

Lemma 1. ([․ Theorem 4.2]) The complementary function of an $N$-function $\Phi$ satisfies the $\Delta_{2}$-condition on $[0,+\infty)$ if there is a constants $l>1$ such that

$$
\Phi(t) \leq \frac{1}{2 l} \Phi(l t) \text { for any } t \geq 0 .
$$

Given an $N$-function $\Phi$, we denote by $L_{\Phi}^{*}$ the collection of all measurable functions $f$ such that $\int_{\mathbb{R}^{n}} \Phi(a f)<\infty$ for some $a>0$.

For a measurable function $f$ on $\mathbb{R}^{2}$, we define the Hardy-Littlewood maximal function of $f$ by setting

$$
M_{f}(x)=\sup f_{B}|f(z)| d z=\sup \frac{1}{|B|} \int_{B}|f(z)| d z,
$$

where the supremum is taken over all open disks $B$ that contain $x$. 
Proposition 2. ([3, Theorem 2.1]) Let $\Phi$ and $\Psi$ be a pair of complementary $N$ functions. The following two conditions are equivalent:

(i) there exists positive constants $C$ and $b$ such that

$$
\int_{\mathbb{R}^{n}} \Phi\left(b M_{f}\right)(z) d z \leq C \int_{\mathbb{R}^{n}} \Phi(|f|)(z) d z, \quad \forall f \in L_{\Phi}^{*},
$$

(ii) $\Psi$ satisfies the $\Delta_{2}$-condition on $[0,+\infty)$.

Example 1. Denote by $\Phi$ the function $\Phi(t)=t^{2} \log ^{\lambda}(e+t)$ for $\lambda>-1$. An elementary computation shows that $\Phi$ is increasing, continuous and convex on $[0,+\infty)$

with $\lim _{t \rightarrow 0+} \frac{\Phi(t)}{t}=0$ and $\lim _{t \rightarrow+\infty} \frac{\Phi(t)}{t}=+\infty$. So $\Phi$ is an $N$-function. Moreover, both the function $\Phi$ and its complementary function satisfy the $\Delta_{2}$-condition. Hence we know that $\Phi$ satisfies Proposition 1 and 2. We will use Proposition 1 frequently in Section 3.1.

Actually, a direct computation shows that the above $\Phi$ satisfies the $\Delta_{2}$-condition on $[0,+\infty)$. In order to check that the complementary $N$-function of $\Phi$ satisfies the $\Delta_{2}$-condition, by Proposition 1, we only need to find a constant $l>1$ so that

$$
2 \log ^{\lambda}(e+t) \leq l \log ^{\lambda}(e+l t), \forall t \geq 0 .
$$

In fact, if $\lambda \geq 0$ we can take $l=2$. By monotonicity of $\log ^{\lambda}(e+\cdot)$, we have that inequality $(2.2)$ holds for any $t \geq 0$. If $-1<\lambda<0$, we take $l=2^{1 /(1+\lambda)}$. By monotonicity we have

$$
\log (e+l t) \leq l \log (e+t), \forall t \geq 0 .
$$

Together with $l=(2 / l)^{1 / \lambda}$, it follows that $(2.2)$ holds for all $t \geq 0$.

\section{Proofs of Theorem 1 and Theorem 2}

In this section, the notation $A \lesssim B$ means that there is a constant $C>0$ so that $A \leq C \cdot B$. Here and in this section, the notation $C$ denotes a positive constant which may differ from line to line. The notation $A \approx B$ means $A \lesssim B$ and $B \lesssim A$.

\subsection{Proof of Theorem 2}

Proof of (iv) $\Leftrightarrow(\mathbf{v})$. Our claim is obvious when $\lambda=0$.

Case $\lambda>0$. Assume (iv) holds. Since $\ell\left(\varphi\left(\Gamma_{j, k}\right)\right) \leq 2 \pi$ for any $\Gamma_{j, k}$ and $\lambda>0$, we have

$$
\log ^{\lambda}\left(e+\frac{\ell\left(\varphi\left(\Gamma_{j, k}\right)\right)}{2^{-j}}\right) \leq \log ^{\lambda}\left(e+2^{j-1} \pi\right) \lesssim j^{\lambda} .
$$


Therefore, we get

$$
\sum_{j=1}^{\infty} \sum_{k=1}^{2^{j}} \ell\left(\varphi\left(\Gamma_{j, k}\right)\right)^{2} \log ^{\lambda}\left(e+\frac{\ell\left(\varphi\left(\Gamma_{j, k}\right)\right)}{2^{-j}}\right) \lesssim \sum_{j=1}^{\infty} j^{\lambda} \sum_{k=1}^{2^{j}} \ell\left(\varphi\left(\Gamma_{j, k}\right)\right)^{2}<+\infty,
$$

which gives the implication (iv) $\Rightarrow(v)$. For the other direction, assume that (v) holds. In order to estimate the logarithmic term from below, we set

$$
\chi(j, k)=\left\{\begin{array}{lc}
1, & \text { if } \quad \ell\left(\varphi\left(\Gamma_{j, k}\right)\right)>2^{-\frac{3 j}{4}} \\
0, & \text { otherwise }
\end{array}\right.
$$

Then

$$
\begin{aligned}
\sum_{j=1}^{\infty} j^{\lambda} \sum_{k=1}^{2^{j}} \ell\left(\varphi\left(\Gamma_{j, k}\right)\right)^{2}= & \sum_{j=1}^{\infty} j^{\lambda} \sum_{k=1}^{2^{j}} \chi(j, k) \ell\left(\varphi\left(\Gamma_{j, k}\right)\right)^{2} \\
& +\sum_{j=1}^{\infty} j^{\lambda} \sum_{k=1}^{2^{j}}(1-\chi(j, k)) \ell\left(\varphi\left(\Gamma_{j, k}\right)\right)^{2}=: P_{1}+P_{2} .
\end{aligned}
$$

If $\ell\left(\varphi\left(\Gamma_{j, k}\right)\right)>2^{-\frac{3 j}{4}}$, then we have

$$
\log ^{\lambda}\left(e+\frac{\ell\left(\varphi\left(\Gamma_{j, k}\right)\right)}{2^{-j}}\right) \geq \log ^{\lambda}\left(e+2^{\frac{1}{4} j}\right) \gtrsim j^{\lambda}
$$

Hence, we obtain from (v) that

$$
P_{1} \lesssim \sum_{j=1}^{\infty} \sum_{k=1}^{2^{j}} \ell\left(\varphi\left(\Gamma_{j, k}\right)\right)^{2} \log ^{\lambda}\left(e+\frac{\ell\left(\varphi\left(\Gamma_{j, k}\right)\right)}{2^{-j}}\right)<+\infty
$$

For $P_{2}$, we have

$$
P_{2} \leq \sum_{j=1}^{\infty} j^{\lambda} \sum_{k=1}^{2^{j}} 2^{-\frac{3}{2} j}=\sum_{j=1}^{+\infty} 2^{-\frac{1}{2} j} j^{\lambda}<+\infty
$$

In conclusion, $P_{1}+P_{2}<+\infty$, and (iv) follows.

Case $\lambda<0$. Assume that (v) holds. Since $\ell\left(\varphi\left(\Gamma_{j, k}\right)\right) \leq 2 \pi$ for any $\Gamma_{j, k}$ and $\lambda<0$, we have

$$
\log ^{\lambda}\left(e+\frac{\ell\left(\varphi\left(\Gamma_{j, k}\right)\right)}{2^{-j}}\right) \geq \log ^{\lambda}\left(e+2^{j-1} \pi\right) \gtrsim j^{\lambda} .
$$

Therefore, we get

$$
\sum_{j=1}^{\infty} j^{\lambda} \sum_{k=1}^{2^{j}} \ell\left(\varphi\left(\Gamma_{j, k}\right)\right)^{2} \lesssim \sum_{j=1}^{\infty} \sum_{k=1}^{2^{j}} \ell\left(\varphi\left(\Gamma_{j, k}\right)\right)^{2} \log ^{\lambda}\left(e+\frac{\ell\left(\varphi\left(\Gamma_{j, k}\right)\right)}{2^{-j}}\right)<+\infty
$$


which gives us the implication $(\mathrm{v}) \Rightarrow(\mathrm{iv})$. For the other direction, assume that (iv) holds. Using the equation 3.1 , if $\ell\left(\varphi\left(\Gamma_{j, k}\right)\right)>2^{-3 j / 4}$, we have

$$
\log ^{\lambda}\left(e+\frac{\ell\left(\varphi\left(\Gamma_{j, k}\right)\right)}{2^{-j}}\right) \leq \log ^{\lambda}\left(e+2^{\frac{1}{4} j}\right) \lesssim j^{\lambda}
$$

Therefore, we obtain

$P_{1}^{\prime}:=\sum_{j=1}^{\infty} \sum_{k=1}^{2^{j}} \chi(j, k) \ell\left(\varphi\left(\Gamma_{j, k}\right)\right)^{2} \log ^{\lambda}\left(e+\frac{\ell\left(\varphi\left(\Gamma_{j, k}\right)\right)}{2^{-j}}\right) \lesssim \sum_{j=1}^{\infty} j^{\lambda} \sum_{k=1}^{2^{j}} \ell\left(\varphi\left(\Gamma_{j, k}\right)\right)^{2}<+\infty$.

Moreover, since $\ell\left(\varphi\left(\Gamma_{j, k}\right)\right) \geq 0$ and $\lambda<0$, we always have

$$
\log ^{\lambda}\left(e+\frac{\ell\left(\varphi\left(\Gamma_{j, k}\right)\right)}{2^{-j}}\right) \leq 1
$$

Hence, we obtain

$$
\begin{aligned}
P_{2}^{\prime} & :=\sum_{j=1}^{\infty} \sum_{k=1}^{2^{j}}(1-\chi(j, k)) \ell\left(\varphi\left(\Gamma_{j, k}\right)\right)^{2} \log ^{\lambda}\left(e+\frac{\ell\left(\varphi\left(\Gamma_{j, k}\right)\right)}{2^{-j}}\right) \\
& \leq \sum_{j=1}^{\infty} \sum_{k=1}^{2^{j}} 2^{-\frac{3}{2} j}=\sum_{j=1}^{\infty} 2^{-\frac{1}{2} j}<+\infty .
\end{aligned}
$$

Then $P_{1}^{\prime}+P_{2}^{\prime}<+\infty$ which gives us $(\mathrm{v})$.

By combining the cases $\lambda=0, \lambda>0$ with $\lambda<0$ above, we finish the proof of (iv) $\Leftrightarrow(\mathrm{v})$.

Proof of (iv) $\Rightarrow(\mathbf{i})$. The harmonic extension of $\varphi$ is given by the Poisson integral formula:

$$
h(z)=\frac{1}{2 \pi} \int_{\mathbb{S}} \frac{1-|z|^{2}}{|z-\zeta|^{2}} \varphi(\zeta)|d \zeta| .
$$

We can therefore compute the $z$-derivative by differentiating this kernel:

$$
h_{z}(z)=\frac{\partial h}{\partial z}(z)=\frac{1}{2 \pi} \int_{\mathbb{S}} \frac{\zeta}{(z-\zeta)^{2}} \varphi(\zeta)|d \zeta|=\frac{1}{2 \pi} \int_{0}^{2 \pi} \frac{e^{i \theta}}{\left(z-e^{i \theta}\right)^{2}} \varphi\left(e^{i \theta}\right) d \theta .
$$

We may write

$$
\varphi\left(e^{i \theta}\right)=\varphi(1) \cdot e^{i f(\theta)}
$$

where $f:[0,2 \pi] \rightarrow[0,2 \pi]$ is continuous and increasing with $f(0)=0$ and $f(2 \pi)=$ $2 \pi$. This allows us to rewrite 3.2 as

$$
h_{z}(z)=\frac{\varphi(1)}{2 \pi i} \int_{0}^{2 \pi} \frac{\partial}{\partial \theta}\left[\frac{1}{\left(z-e^{i \theta}\right)}\right] e^{i f(\theta)} d \theta=\frac{\varphi(1)}{2 \pi i} \int_{0}^{2 \pi} e^{i f(\theta)} d\left(\frac{1}{\left(z-e^{i \theta}\right)}\right) .
$$


Here we use the Lebesgue-Stieltjes integration on the right-hand side of (3.3). By integrating the right-hand side of (3.3) by parts, we obtain the estimate

$$
h_{z}(z)=\frac{\varphi(1)}{2 \pi i} \int_{0}^{2 \pi} \frac{1}{\left(z-e^{i \theta}\right)} d\left(e^{i f(\theta)}\right) .
$$

We therefore have

$$
\left|h_{z}(z)\right| \leq \frac{1}{2 \pi} \int_{0}^{2 \pi} \frac{1}{\mid z-e^{i \theta \mid}}\left|d\left(e^{i f(\theta)}\right)\right| .
$$

Denote by $\mu_{f}$ the Lebesgue-Stieltjes measure of the continuous increasing function $f$. Then we have $\left|d\left(e^{i f(\theta)}\right)\right| \leq d(f(\theta))=d \mu_{f}(\theta)$. Therefore, we obtain the formula

$$
\left|h_{z}(z)\right| \leq \frac{1}{2 \pi} \int_{0}^{2 \pi} \frac{1}{\mid z-e^{i \theta \mid}} d \mu_{f}(\theta) .
$$

Using our decomposition of the unit disk $\mathbb{D}$ and the decomposition $\mathcal{P}\left(I_{j, k}\right)$ of $[0,2 \pi]$ in Section 2.1. for $\Phi(t)=t^{2} \log ^{\lambda}(e+t)$, we get

$$
\begin{aligned}
\int_{\mathbb{D}} \Phi\left(\left|h_{z}(z)\right|\right) d z & =\sum_{j=1}^{+\infty} \sum_{k=1}^{2^{j}} \int_{Q_{j, k}} \Phi\left(\left|h_{z}(z)\right|\right) d z \\
& \leq \sum_{j=1}^{+\infty} \sum_{k=1}^{2^{j}} \int_{Q_{j, k}} \Phi\left(\frac{1}{2 \pi} \sum_{I \in \mathcal{P}\left(I_{j, k}\right)} \int_{I} \frac{1}{\mid z-e^{i \theta \mid}} d \mu_{f}(\theta)\right) d z \\
& =\sum_{j=1}^{+\infty} \sum_{k=1}^{2^{j}} \int_{Q_{j, k}} \Phi\left(\frac{1}{2 \pi} \sum_{n \leq j} \sum_{m} \int_{I_{n, m}} \frac{1}{\mid z-e^{i \theta \mid}} d \mu_{f}(\theta)\right) d z
\end{aligned}
$$

where we abuse the notation: sum over those $m$ which belong to $\left\{1, \cdots, 2^{n}\right\}$ and satisfy $I_{n, m} \in \mathcal{P}\left(I_{j, k}\right)$. It is easy to check that $\#\{m\} \leq 3$. For any $I_{n, m} \in \mathcal{P}\left(I_{j, k}\right)$, we know that $\left|z-e^{i \theta}\right| \approx 2^{-n}$ for any $z \in Q_{j, k}$ and $\theta \in I_{n, m}$. Since $\mu_{f}$ is the LebesgueStieltjes measure of our continuous increasing function $f$, for any interval $I_{n, m}$, we get

$$
\int_{I_{n, m}} d \mu_{f}(\theta)=\left|f\left(I_{n, m}\right)\right|=\ell\left(\varphi\left(\Gamma_{n, m}\right)\right) .
$$

Hence we obtain

$$
\begin{aligned}
\int_{\mathbb{D}} \Phi\left(\left|h_{z}(z)\right|\right) d z & \leq \sum_{j=1}^{+\infty} \sum_{k=1}^{2^{j}} \int_{Q_{j, k}} \Phi\left(\sum_{n \leq j} \sum_{m} \frac{\ell\left(\varphi\left(\Gamma_{n, m}\right)\right)}{2^{-n}}\right) d z \\
& \lesssim \sum_{j=1}^{+\infty} \sum_{k=1}^{2^{j}} 2^{-2 j} \Phi\left(\sum_{n \leq j} \sum_{m} \frac{\ell\left(\varphi\left(\Gamma_{n, m}\right)\right)}{2^{-n}}\right) .
\end{aligned}
$$


Since $\ell\left(\varphi\left(\Gamma_{n, m}\right)\right) \leq 2 \pi$, we have

$$
\sum_{n \leq j} \sum_{m} \frac{\ell\left(\varphi\left(\Gamma_{n, m}\right)\right)}{2^{-n}} \leq 2 \pi \sum_{n \leq j} \sum_{m} \frac{1}{2^{-n}} \lesssim 2^{j} .
$$

Using the same idea as in Proof of (iv) $\Leftrightarrow(\mathrm{v})$, we have the estimate

$$
\sum_{j=1}^{+\infty} \sum_{k=1}^{2^{j}} 2^{-2 j} \Phi\left(\sum_{n \leq j} \sum_{m} \frac{\ell\left(\varphi\left(\Gamma_{n, m}\right)\right)}{2^{-n}}\right) \lesssim \sum_{j=1}^{+\infty} \sum_{k=1}^{2^{j}} 2^{-2 j} j^{\lambda}\left(\sum_{n \leq j} \sum_{m} \frac{\ell\left(\varphi\left(\Gamma_{n, m}\right)\right)}{2^{-n}}\right)^{2}+C .
$$

This allows us to estimate $(3.6)$ as

$$
\int_{\mathbb{D}} \Phi\left(\left|h_{z}(z)\right|\right) d z \lesssim \sum_{j=1}^{+\infty} \sum_{k=1}^{2^{j}} 2^{-2 j} j^{\lambda}\left(\sum_{n \leq j} \sum_{m} \frac{\ell\left(\varphi\left(\Gamma_{n, m}\right)\right)}{2^{-n}}\right)^{2}+C .
$$

For fixed $\varphi\left(\Gamma_{n, m}\right)$ and fixed $j \geq n$, the estimate (2.1) gives us $\#\{k\} \leq 3 \cdot 2^{j-n}$. By applying the Hölder inequality on the right-hand side of inequality (3.7) and Fubini's theorem for series, we obtain

$$
\begin{aligned}
\int_{\mathbb{D}} \Phi\left(\left|h_{z}(z)\right|\right) d z & \lesssim \sum_{j=1}^{+\infty} \sum_{k=1}^{2^{j}} 2^{-\frac{3}{2} j} j^{\lambda} \sum_{n \leq j} \sum_{m} \frac{\ell\left(\varphi\left(\Gamma_{n, m}\right)\right)^{2}}{2^{-\frac{3}{2} n}}+C \\
& \lesssim \sum_{n=1}^{+\infty} \sum_{m=1}^{2^{n}} \frac{\ell\left(\varphi\left(\Gamma_{n, m}\right)\right)^{2}}{2^{-\frac{3}{2} n}}\left(\sum_{j \geq n} \sum_{k} 2^{-\frac{3}{2} j} j^{\lambda}\right)+C \\
& \lesssim \sum_{n=1}^{+\infty} \sum_{m=1}^{2^{n}} \frac{\ell\left(\varphi\left(\Gamma_{n, m}\right)\right)^{2}}{2^{-\frac{1}{2} n}} \underbrace{\left(\sum_{j \geq n} 2^{-\frac{1}{2} j} j^{\lambda}\right)}_{\approx 2^{-\frac{1}{2} n} n^{\lambda}}+C \\
& \lesssim \sum_{n=1}^{+\infty} \sum_{m=1}^{2^{n}} n^{\lambda} \ell\left(\varphi\left(\Gamma_{n, m}\right)\right)^{2}+C<+\infty
\end{aligned}
$$

An analogous estimate follows for $h_{\bar{z}}$ by the same reasons. Thus we finish the proof of (iv) $\Rightarrow$ (i).

Proof of (iv) $\Rightarrow$ (ii). Recall the estimate (3.4) for $\left|h_{z}\right|$ and formula (3.5). From (3.5) and the fact that $\log ^{\lambda}\left(\frac{2}{1-|z|}\right) \approx j^{\lambda}$ for any $z \in Q_{j, k}$, we have

$$
\int_{\mathbb{D}}\left|h_{z}(z)\right|^{2} \log ^{\lambda}\left(\frac{2}{1-|z|}\right) d z=\sum_{j=1}^{+\infty} \sum_{k=1}^{2^{j}} \int_{Q_{j, k}}\left|h_{z}(z)\right|^{2} \log ^{\lambda}\left(\frac{2}{1-|z|}\right) d z
$$




$$
\approx \sum_{j=1}^{+\infty} \sum_{k=1}^{2^{j}} j^{\lambda} \int_{Q_{j, k}}\left|h_{z}(z)\right|^{2} d z
$$

By replacing $\Phi(t)=t^{2} \log ^{\lambda}(e+t)$ by $t^{2}$ in $(3.6)$, we obtain

$$
\int_{Q_{j, k}}\left|h_{z}(z)\right|^{2} d z \lesssim 2^{-2 j}\left(\sum_{n \leq j} \sum_{m} \frac{\ell\left(\varphi\left(\Gamma_{n, m}\right)\right)}{2^{-n}}\right)^{2}
$$

Hence we have

$$
\int_{\mathbb{D}}\left|h_{z}(z)\right|^{2} \log ^{\lambda}\left(\frac{2}{1-|z|}\right) d z \lesssim \sum_{j=1}^{+\infty} \sum_{k=1}^{2^{j}} 2^{-2 j} j^{\lambda}\left(\sum_{n \leq j} \sum_{m} \frac{\ell\left(\varphi\left(\Gamma_{n, m}\right)\right)}{2^{-n}}\right)^{2} .
$$

Consequently, we may apply the very same arguments that we used above to estimate the right-hand side of (3.7) so as to arrive at

$$
\int_{\mathbb{D}}\left|h_{z}(z)\right|^{2} \log ^{\lambda}\left(\frac{2}{1-|z|}\right) d z \lesssim \sum_{n=1}^{+\infty} \sum_{m=1}^{2^{n}} n^{\lambda} \ell\left(\varphi\left(\Gamma_{n, m}\right)\right)^{2}<+\infty
$$

One may similarly deal with $h_{\bar{z}}$ to finish the proof of (iv) $\Rightarrow$ (ii).

Proof of (iv) $\Leftrightarrow($ iii). We first divide the double integral on $\mathbb{S}$ into two parts:

$$
\begin{aligned}
& \int_{\mathbb{S}} \int_{\mathbb{S}}|\log | \varphi^{-1}(\xi)-\varphi^{-1}(\eta)||^{\lambda+1}|d \eta||d \xi| \\
= & \int_{\mathbb{S}} \int_{\left\{\eta \in \mathbb{S}: 1 \leq\left|\varphi^{-1}(\xi)-\varphi^{-1}(\eta)\right| \leq 2\right\}}+\int_{\mathbb{S}} \int_{\left\{\eta \in \mathbb{S}: 0 \leq\left|\varphi^{-1}(\xi)-\varphi^{-1}(\eta)\right|<1\right\}}=I+I I .
\end{aligned}
$$

Since $\lambda>-1$, we have $0 \leq|\log t|^{\lambda+1} \leq(\log 2)^{\lambda+1}$ for $1 \leq t \leq 2$. Therefore we obtain $0 \leq I \leq C$. So our job is to estimate $I I$.

Fubini's theorem changes $I I$ to

$$
\begin{aligned}
I I & =\int_{\mathbb{S}} \int_{\left\{\eta \in \mathbb{S}:\left|\varphi^{-1}(\xi)-\varphi^{-1}(\eta)\right|<1\right\}} \int_{0}^{1} \Lambda(t) \chi_{\left\{\left|\varphi^{-1}(\xi)-\varphi^{-1}(\eta)\right| \leq t<1\right\}} d t|d \eta||d \xi| \\
& =\int_{\mathbb{S}} \int_{0}^{1} \ell\left(\left\{\eta \in \mathbb{S}:\left|\varphi^{-1}(\xi)-\varphi^{-1}(\eta)\right| \leq t\right\}\right) \Lambda(t) d t|d \xi|
\end{aligned}
$$

where $\Lambda(t)=(\lambda+1) \log ^{\lambda}\left(\frac{1}{t}\right) / t$. Here we used the fact that

$$
|\log t|^{\lambda+1}=\log ^{\lambda+1}\left(\frac{1}{t}\right)=\int_{t}^{1} \Lambda(t) d t, \text { for any } 0<t<1 .
$$


Then we have

$$
\begin{aligned}
I I & =\sum_{j=0}^{+\infty} \int_{\mathbb{S}} \int_{2^{-j-1}}^{2^{-j}} \ell\left(\left\{\eta:\left|\varphi^{-1}(\xi)-\varphi^{-1}(\eta)\right| \leq t\right\}\right) \Lambda(t) d \xi d t \\
& \leq C+\sum_{j=1}^{+\infty} \int_{\mathbb{S}} \ell\left(\left\{\eta:\left|\varphi^{-1}(\xi)-\varphi^{-1}(\eta)\right| \leq 2^{-j}\right\}\right)|d \xi| \int_{2^{-j-1}}^{2^{-j}} \Lambda(t) d t
\end{aligned}
$$

If $\xi \in \varphi\left(\Gamma_{j, k}\right)$ and $\left|h^{-1}(\xi)-h^{-1}(\eta)\right| \leq 2^{-j}$, then the arc length of the shorter arc from $h^{-1}(\xi)$ to $h^{-1}(\eta)$ is at most $2^{-j} \pi$ and hence it follows that $\eta \in \cup_{n=k-1}^{k+1} \varphi\left(\Gamma_{j, n}\right)$. This means that for $j \geq 1$, we have

$$
\begin{aligned}
& \sum_{k=1}^{2^{j}} \int_{\varphi\left(\Gamma_{j, k}\right)} \ell\left(\left\{\eta:\left|\varphi^{-1}(\xi)-\varphi^{-1}(\eta)\right| \leq 2^{-j}\right\}\right)|d \xi| \\
& \quad \leq \sum_{k=1}^{2^{j}} \ell\left(\cup_{n=k-1}^{k+1} \varphi\left(\Gamma_{j, n}\right)\right) \ell\left(\varphi\left(\Gamma_{j, k}\right) \lesssim \sum_{k=1}^{2^{j}} \ell\left(\varphi\left(\Gamma_{j, k}\right)\right)^{2} .\right.
\end{aligned}
$$

By the mean value theorem, we have

$$
\int_{2^{-j-1}}^{2^{-j}} \Lambda(t) d t=\log ^{\lambda+1}\left(2^{j+1}\right)-\log ^{\lambda+1}\left(2^{j}\right) \approx j^{\lambda}, \quad j \geq 1 .
$$

Combining (3.8), 3.9), 3.10, 3.11) and (3.12), we therefore deduce

$$
\int_{\mathbb{S}} \int_{\mathbb{S}}|\log | \varphi^{-1}(\xi)-\left.\varphi^{-1}(\eta)\right|^{\lambda+1}|d \xi||d \eta| \lesssim \sum_{j=1}^{+\infty} \sum_{k=1}^{2^{j}} \ell\left(\varphi\left(\Gamma_{j, k}\right)\right)^{2} j^{\lambda}+C .
$$

We are left to deal with the converse direction. We divide the interval $[0,1]$ in (3.9) into intervals $[\pi / 8,1]$ and $\left[2^{-j+1} \pi, 2^{-j+2} \pi\right]$ for $j \geq 5$. Then we have

$$
I I \geq \sum_{j=5}^{+\infty} \int_{\mathbb{S}} \ell\left(\left\{\eta:\left|\varphi^{-1}(\xi)-\varphi^{-1}(\eta)\right| \leq 2^{-j+1} \pi\right\}\right)|d \xi| \int_{2^{-j+1} \pi}^{2^{-j+2} \pi} \Lambda(t) d t
$$

Given any $j \geq 5$ and $1 \leq k \leq 2^{j}$, the inequality

$$
\left|\varphi^{-1}(\xi)-\varphi^{-1}(\eta)\right| \leq \ell\left(\Gamma_{j, k}\right) \leq \pi 2^{1-j}
$$

holds for all $\eta, \xi \in \varphi\left(\Gamma_{j, k}\right)$. Thus we have

$$
\sum_{k=1}^{2^{j}} \int_{\varphi\left(\Gamma_{j, k}\right)} \ell\left(\left\{\eta:\left|h^{-1}(\xi)-h^{-1}(\eta)\right| \leq \pi 2^{1-j}\right\}\right)|d \xi| \geq \sum_{k=1}^{2^{j}} \ell\left(\varphi\left(\Gamma_{j, k}\right)\right)^{2}
$$


Finally, the estimates (3.8), 3.12, 3.13) and 3.14 yield

$$
\int_{\mathbb{S}} \int_{\mathbb{S}}|\log | \varphi^{-1}(\xi)-\left.\varphi^{-1}(\eta)\right|^{\lambda+1}|d \xi||d \eta|+C \gtrsim \sum_{j=1}^{+\infty} \sum_{k=1}^{2^{j}} \ell\left(\varphi\left(\Gamma_{j, k}\right)\right)^{2} j^{\lambda} .
$$

Proof of (ii) $\Rightarrow$ (iv). Since $\varphi$ is uniformly continuous, there exists $j_{0} \geq 3$ such that $\ell\left(\varphi\left(\Gamma_{j, k}\right)\right) \leq \pi / 3$ whenever $j \geq j_{0}$ and $k=1, \cdots, 2^{j}$. Fix such $j, k$ and let $\xi \in \Gamma_{j, k-1}$, $\eta \in \Gamma_{j, k+1}\left(\Gamma_{j, 0}=\Gamma_{j, 2^{j}}, \Gamma_{j, 2^{j}+1}=\Gamma_{j, 1}\right)$. Since $h \in C(\overline{\mathbb{D}})$ is a diffeomorphism, we have that

$$
|h(\xi)-h(\eta)| \leq \int_{\gamma}|D h| d s .
$$

for any rectifiable curve $\gamma \subset \mathbb{D}$ joining $\xi$ to $\eta$. Moreover, $\left|h\left(\xi_{j, k}\right)-h\left(\xi_{j, k+1}\right)\right| \leq \mid h(\xi)-$ $h(\eta) \mid$ because $\varphi$ is homeomorphic and $\ell\left(\varphi\left(\Gamma_{j, k-1} \cup \Gamma_{j, k} \cup \Gamma_{j, k+1}\right)\right) \leq \pi$. Denote by $\hat{\xi}$ the midpoint of the arc $\Gamma_{j, k}$. Let $t_{1}>0$ be the smallest $t$ for which $\partial B(\hat{\xi}, t) \cap \Gamma_{j, k+1} \neq \emptyset$ and let $t_{2}$ be correspondingly the largest such $t$. Write $\gamma_{t}=\partial B(\hat{\xi}, t) \cap \mathbb{D}$ for $t_{1} \leq$ $t \leq t_{2}$. Then there exists an absolute constant $C$ so that

$$
\bigcup_{t \in\left[t_{1}, t_{2}\right]} \gamma_{t} \subset C Q_{j, k} \cap \mathbb{D}
$$

Now

$$
\left|h\left(\xi_{j, k}\right)-h\left(\xi_{j, k+1}\right)\right| \leq \int_{\gamma_{t}}|D h| d s
$$

for $t \in\left[t_{1}, t_{2}\right]$ and by integrating with respect to $t$ we obtain

$$
\left(t_{2}-t_{1}\right)\left|h\left(\xi_{j, k}\right)-h\left(\xi_{j, k+1}\right)\right| \leq \int_{t_{1}}^{t_{2}} \int_{\gamma_{t}}|D h| d s d t \leq \int_{C Q_{j, k} \cap \mathbb{D}}|D h(z)| d z .
$$

Notice that $t_{2}-t_{1}$ is uniformly comparable to $2^{-j} \approx \ell\left(\Gamma_{j, k}\right)$ when $j \geq j_{0}$ and $k \in\left\{1, \cdots, 2^{j}\right\}$. It follows from 3.15 that

$$
\left|h\left(\xi_{j, k}\right)-h\left(\xi_{j, k+1}\right)\right| \lesssim \frac{1}{\ell\left(\Gamma_{j, k}\right)} \int_{C Q_{j, k} \cap \mathbb{D}}|D h(z)| d z
$$

Recall that we have $\ell\left(\varphi\left(\Gamma_{j, k}\right)\right) \leq \pi / 3$. Thus $\ell\left(\varphi\left(\Gamma_{j, k}\right)\right)$ is also uniformly comparable to $\left|\varphi\left(\xi_{j, k}\right)-\varphi\left(\xi_{j, k+1}\right)\right|$. We can therefore conclude from (3.16) that for any $j \geq j_{0}$ and $1 \leq k \leq 2^{j}$, the following inequality holds:

$$
\ell\left(\varphi\left(\Gamma_{j, k}\right)\right) \lesssim \frac{1}{\ell\left(\Gamma_{j, k}\right)} \int_{C Q_{j, k} \cap \mathbb{D}}|D h(z)| d z
$$


Fix $p \in(1,2)$ and $q \in(2,+\infty)$ with $1 / p+1 / q=1$. By applying the Hölder inequality to (3.17), we have

$$
\ell\left(\varphi\left(\Gamma_{j, k}\right)\right)^{p} \lesssim \frac{1}{\ell\left(\Gamma_{j, k}\right)^{p}} \int_{C Q_{j, k}} H(z) d z\left(\int_{C Q_{j, k} \cap \mathbb{D}} \log ^{-\frac{\lambda q}{2}}\left(\frac{1}{1-|z|}\right) d z\right)^{p / q},
$$

where $H(z)=|D h(z)|^{p} \log ^{\lambda p / 2}\left(\frac{1}{1-|z|}\right) \chi_{\mathbb{D}}(z)$. Moreover by changing to polar coordinates we have the estimate

$$
\begin{aligned}
\int_{C Q_{j, k} \cap \mathbb{D}} \log ^{-\frac{\lambda q}{2}}\left(\frac{1}{1-|z|}\right) d z & \lesssim \ell\left(\Gamma_{j, k}\right) \int_{0}^{C \ell\left(\Gamma_{j, k}\right)} \log ^{-\frac{\lambda q}{2}}\left(\frac{1}{s}\right) d s \\
& \lesssim \ell\left(\Gamma_{j, k}\right)^{2} \log ^{-\frac{\lambda q}{2}}\left(\frac{1}{\ell\left(\Gamma_{j, k}\right)}\right) \\
& \approx \ell\left(\Gamma_{j, k}\right)^{2} j^{-\lambda q / 2} .
\end{aligned}
$$

It follows that

$$
\ell\left(\varphi\left(\Gamma_{j, k}\right)\right)^{p} j^{\lambda p / 2} \lesssim \ell\left(\Gamma_{j, k}\right)^{p} f_{C Q_{j, k} \cap \mathbb{D}} H(z) d z .
$$

Next by the inclusion relationship between $B_{j, k}$ and $Q_{j, k}$ and the definition of HardyLitterwood maximal function we have

$$
f_{C Q_{j, k}} H(z) d z \leq f_{C B_{j, k}} H(z) d z \leq f_{B_{j, k}} M_{H}(z) d z \leq f_{Q_{j, k}} M_{H}(z) d z .
$$

Combining (3.18) with (3.19) and then applying Jensen's inequality, we arrive at

$$
\ell\left(\varphi\left(\Gamma_{j, k}\right)\right)^{2} j^{\lambda} \lesssim \int_{Q_{j, k}} M_{H}^{2 / p}(z) d z, \quad \forall j \geq j_{0} \text { and } 1 \leq k \leq 2^{j} .
$$

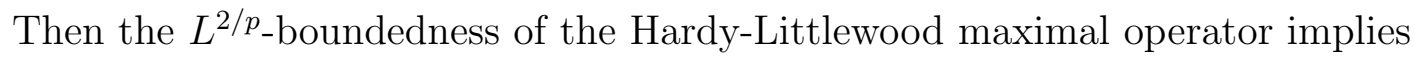

$$
\begin{aligned}
\sum_{j=j_{0}}^{+\infty} \sum_{k=1}^{2^{j}} \ell\left(\varphi\left(\Gamma_{j, k}\right)\right)^{2} j^{\lambda} & \lesssim \sum_{j=1}^{+\infty} \sum_{k=1}^{2^{j}} \int_{Q_{j, k}} M_{H}^{2 / p}(z) d z \leq \int_{\mathbb{R}^{2}} M_{H}^{2 / p}(z) d z \\
& \lesssim \int_{\mathbb{D}}|D h(z)|^{2} \log ^{\lambda}\left(\frac{1}{1-|z|}\right) d z .
\end{aligned}
$$

Moreover, we have that

$$
\sum_{j=1}^{j_{0}-1} \sum_{k=1}^{2^{j}} \ell\left(\varphi\left(\Gamma_{j, k}\right)\right)^{2} j^{\lambda} \leq \sum_{j=1}^{j_{0}-1} j^{\lambda}\left(\sum_{k=1}^{2^{j}} \ell\left(\varphi\left(\Gamma_{j, k}\right)\right)\right)^{2} \lesssim \sum_{j=1}^{j_{0}-1} j^{\lambda} \leq C .
$$


Hence we conclude

$$
\begin{aligned}
\sum_{j=0}^{+\infty} \sum_{k=1}^{2^{j}} \ell\left(\varphi\left(\Gamma_{j, k}\right)\right)^{2} j^{\lambda} & =\sum_{j=1}^{j_{0}-1} \sum_{k=1}^{2^{j}} \ell\left(\varphi\left(\Gamma_{j, k}\right)\right)^{2} j^{\lambda}+\sum_{j=j_{0}}^{+\infty} \sum_{k=1}^{2^{j}} \ell\left(\varphi\left(\Gamma_{j, k}\right)\right)^{2} j^{\lambda} \\
& \lesssim C+\int_{\mathbb{D}}|D h(z)|^{2} \log ^{\lambda}\left(\frac{1}{1-|z|}\right) d z .
\end{aligned}
$$

Proof of $(\mathbf{i}) \Rightarrow(\mathbf{v})$. Set $K(z)=|D h(z)| \chi_{\mathbb{D}}(z)$. As in the proof of (ii) $\Rightarrow$ (iv), we have

$$
\frac{\ell\left(\varphi\left(\Gamma_{j, k}\right)\right)}{\ell\left(\Gamma_{j, k}\right)} \lesssim f_{C Q_{j, k}} K(z) d z \text { for any } j \geq j_{0} \text { and } 1 \leq k \leq 2^{j}
$$

Thus Jensen's inequality for the function $\Phi(t)=t^{2} \log ^{\lambda}(e+t)$ and inequality 3.19 with $H$ replaced by $K$ imply that

$$
\begin{aligned}
\sum_{j=j_{0}}^{+\infty} \sum_{k=1}^{2^{j}} \ell\left(\Gamma_{j, k}\right)^{2} \Phi\left(\frac{\ell\left(\phi\left(\Gamma_{j, k}\right)\right)}{\ell\left(\Gamma_{j, k}\right)}\right) & \lesssim \sum_{j=1}^{+\infty} \sum_{k=1}^{2^{j}} \ell\left(\Gamma_{j, k}\right)^{2} f_{Q_{j, k}} \Phi\left(M_{K}\right)(z) d z \\
& \lesssim \sum_{j=1}^{+\infty} \sum_{k=1}^{2^{j}} \int_{Q_{j, k}} \Phi\left(M_{K}\right)(z) d z \leq \int_{\mathbb{R}^{2}} \Phi\left(M_{K}\right)(z) d z
\end{aligned}
$$

Here in the second inequality, we used the fact that $\ell\left(\Gamma_{j, k}\right)^{2} \approx\left|Q_{j, k}\right|$. Therefore Proposition 2 gives

$$
\int_{\mathbb{R}^{2}} \Phi\left(M_{K}\right)(z) d z \lesssim \int_{\mathbb{R}^{2}} \Phi(K)(z) d z
$$

Moreover, we have that

$$
\sum_{j=1}^{j_{0}-1} \sum_{k=1}^{2^{j}} \ell\left(\varphi\left(\Gamma_{j, k}\right)\right)^{2} \log ^{\lambda}\left(e+\frac{\ell\left(\varphi\left(\Gamma_{j, k}\right)\right)}{\ell\left(\Gamma_{j, k}\right)}\right) \lesssim \sum_{j=1}^{j_{0}-1} \sum_{k=1}^{2^{j}} \ell\left(\varphi\left(\Gamma_{j, k}\right)\right)^{2} j^{\lambda} \lesssim \sum_{j=1}^{j_{0}-1} j^{\lambda} \leq C
$$

Combining (3.20, 3.21) with the above inequality gives

$$
\sum_{j=1}^{+\infty} \sum_{k=1}^{2^{j}} \ell\left(\varphi\left(\Gamma_{j, k}\right)\right)^{2} \log ^{\lambda}\left(e+\frac{\ell\left(\varphi\left(\Gamma_{j, k}\right)\right)}{\ell\left(\Gamma_{j, k}\right)}\right) \lesssim C+\int_{\mathbb{D}}|D h(z)|^{2} \log ^{\lambda}(e+|D h(z)|) d z
$$




\subsection{Proof of Theorem 1}

Proof. From [2, Lemma 3.2], we know that any internal chord-arc Jordan domain $\Omega \subset \mathbb{C}$ is a bounded John disk whose boundary $\partial \Omega$ satisfies $(1.1)$. Note also that in bounded John disks, the internal distance of any two boundary points is finite, [9, Remark 6.6]. By using the arc length parametrization of $\partial \Omega$ and the property (1.1), we see that there is a bi-Lipschitz map $g: \mathbb{S} \rightarrow\left(\partial \Omega, \lambda_{\Omega}\right)$. Then applying [2, Theorem 4.7] and the fact that the internal distance in the unit disk is the same as the Euclidean distance, we know that $g$ extends to a bi-Lipschitz map $\tilde{g}: \overline{\mathbb{D}} \rightarrow\left(\bar{\Omega}, \lambda_{\Omega}\right)$. Moreover, the bi-Lipschitz map $\tilde{g}$ is a diffeomorphism in $\mathbb{D}$. Indeed, by the construction of the map $\tilde{g}$ in [2, Theorem 4.7], $\tilde{g}=\psi_{1} \circ \tilde{f}$, where $\psi_{1}: \mathbb{D} \rightarrow \Omega$ is a conformal map and $\tilde{f}: \overline{\mathbb{D}} \rightarrow \overline{\mathbb{D}}$ is a homeomorphic extension of $f=\psi_{1}^{-1} \circ g$ from [5, Lemma 2.10]. By the construction of $\tilde{f}$ in [5, Lemma 2.10], $\tilde{f}=\psi_{2}^{-1} \circ \bar{f} \circ \psi_{2}$, where $\psi_{2}: \overline{\mathbb{D}} \rightarrow \overline{\mathbb{H}}^{2}$ is a Möbius map and $\bar{f}: \bar{H}^{2} \rightarrow \overline{\mathbb{H}}^{2}$ is the Beurling-Ahlfors extension of the quasisymmetric map $\psi_{2} \circ f \circ \psi_{2}^{-1}$. Since conformal maps, Möbius maps and Beurling-Ahlfors extensions of quasisymmetric maps are diffeomorphic (cf. [4, Chapter 8]), we obtain that the map $\tilde{g}$ is a diffeomorphism.

In the statements (i) and (ii) in Theorem 1, we let $h=\tilde{g} \circ P\left[g^{-1} \circ \varphi\right]$, where $P\left[g^{-1} \circ \varphi\right]$ is the Poisson extension of $g^{-1} \circ \varphi$. Hence $h$ is a diffeomorphism. Since $\tilde{g}$ is bi-Lipschitz with respect to the internal distance and the internal distance $\lambda_{\Omega}$ is the same as the Euclidean distance locally in $\Omega$, we obtain that there is a constant $C>0$ so that $1 / C \leq|D \tilde{g}(z)| \leq C$ for any $z \in \mathbb{D}$. Hence the convergence of the integrals of $|D h|$ in (i) and (ii) in Theorem 1 is equivalent to the statements (i) and (ii) for $P\left[g^{-1} \circ \varphi\right]$ in Theorem 2 .

By [2, Lemma 3.2, Lemma 3.4 and Lemma 3.6], we know that for any $z_{1}, z_{2} \in \partial \Omega$, the shorter arc $\gamma_{z_{1}, z_{2}}$ of $\partial \Omega$ joining $z_{1}$ and $z_{2}$ satisfies

$$
\operatorname{diam}\left(\gamma_{z_{1}, z_{2}}\right) \approx \lambda_{\Omega}\left(z_{1}, z_{2}\right)
$$

Hence the arc length $\ell(\cdot)$ on $\partial \Omega$ with respect to the Euclidean distance is comparable to the arc length $\ell_{\lambda_{\Omega}}(\cdot)$ on $\partial \Omega$ with respect to the internal distance $\lambda_{\Omega}$. Combining with the bi-Lipschitz property of $g$, we obtain that $\ell\left(\varphi\left(\Gamma_{j, k}\right)\right) / C \leq \ell\left(g^{-1} \circ \varphi\left(\Gamma_{j, k}\right)\right) \leq$ $C \ell\left(\varphi\left(\Gamma_{j, k}\right)\right)$ for any $\Gamma_{j, k}$ and that we can use a change of variable via $g$ to conclude the equivalence of (iii) for $\varphi^{-1}$ and $\varphi^{-1} \circ g$ in Theorem 1 and 2 . Hence the statements (iii)-(v) for $\varphi$ in Theorem 1 is equivalent to the statements (iii)-(v) for $g^{-1} \circ \varphi$ in Theorem 2 .

\section{Acknowledgment}

The first and second authors have been supported by the Academy of Finland via Centre of Excellence in Analysis and Dynamics Research (project No. 307333). The third author has been supported by the graduate school of University of Science 
and Technology of China, and by China Scholarship Council. This work was finished when the third author visited Department of Mathematics and Statistics at University of Jyväskylä. He thanks the department for the hospitality.

\section{References}

[1] K. Astala, T. Iwaniec, G. J. Martin, and J. Onninen: Extremal mappings of finite distortion, Proc. London Math. Soc. 91 (2005), no. 3, 655-702.

[2] O. J. Broch: Extension of internally bilipschitz maps in John disks. Ann. Acad. Sci. Fenn. Math. Volumen 31, (2006), 13-31.

[3] D. Gallardo: Orlicz spaces for which the Hardy-Littlewood maximal operator is bounded. Publ. Mat. 32 (1988), no. 2, 261-266.

[4] F. W. Gehring, G. J. Martin, and B. P. Palka: An introduction to the theory of higher-dimensional quasiconformal mappings. Mathematical Surveys and Monographs, 216. American Mathematical Society, Providence, RI, 2017, page 355 .

[5] M. Ghamsari, R. Näkki, and J. Väisälä: John disks and extension of maps. Monatsh. Math. 117, (1994), 63-94.

[6] T. Iwaniec, G. Martin, and C. Sbordone: $L^{p}$-integrability \& weak type $L^{2}$-estimates for the gradient of harmonic mappings of $\mathbb{D}$. Discrete Contin. Dyn. Syst. Ser. B 11 (2009), no. 1, 145-152.

[7] H. Kneser: Lösung der Aufgabe 41, Jahresber. Deutsch. Math.-Verein. 35 (1926), 123-124.

[8] M. A. Krasnosel'skij and Ya. B. Rutitskij, Convex functions and Orlicz spaces, Fizmatgiz, Moscow 1958, 271 pp.; English transl., P. Noordhoff Ltd., Groningen 1961, xi+249 pp.

[9] R. Näkki, and J. Väisälä: John disks. Expo. Math. 9, (1991), 9-43.

[10] G. C. Verchota: Harmonic homeomorphisms of the closed disc to itself need be in $W^{1, p}, p<2$, but not $W^{1,2}$. Proc. Amer. Math. Soc. 135 (2007), no. 3, 891-894.

[11] H. Xu: Weighted estimates for Poisson extensions of homeomorphisms, in preparation. 
Pekka Koskela

Department of Mathematics and Statistics, University of Jyväskylä, PO Box 35, FI-40014 Jyväskylä, Finland

E-mail address: pekka.j.koskela@jyu.fi

Zhuang Wang

Department of Mathematics and Statistics, University of Jyväskylä, PO Box 35, FI-40014 Jyväskylä, Finland

E-mail address: zhuang.z.wang@jyu.fi

Haiqing Xu

School of Mathematical Sciences, University of Science and Technology of China, Hefei 230026, P. R. China

E-mail address: hqxu@mail.ustc.edu.cn 